\title{
Literature Review of the Use of Zinc and Zinc Compounds in Paper-Based Microfluidic Devices
}

\author{
Amer Charbaji, Hojat Heidari-Bafroui, Constantine Anagnostopoulos, Mohammad Faghri \\ Microfluidics Laboratory, Department of Mechanical, Industrial and Systems Engineering, University of Rhode Island, Kingston, \\ USA \\ Email: charbaji@uri.edu, faghrim@uri.edu
}

How to cite this paper: Charbaji, A., Heidari-Bafroui, H., Anagnostopoulos, C. and Faghri, M. (2021) Literature Review of the Use of Zinc and Zinc Compounds in Paper-Based Microfluidic Devices. Journal of Minerals and Materials Characterization and Engineering, 9, 257-270.

https://doi.org/10.4236/jmmce.2021.93018

Received: March 29, 2021

Accepted: May 11, 2021

Published: May 14, 2021

Copyright $\odot 2021$ by author(s) and Scientific Research Publishing Inc. This work is licensed under the Creative Commons Attribution International License (CC BY 4.0).

http://creativecommons.org/licenses/by/4.0/

\begin{abstract}
Zinc and its compounds, alloys and composites play an important role in the modern day world and find application in almost every aspect that can improve the quality of our lives. This ranges from supplements and pharmaceuticals that are meant to improve our health and wellbeing to additives meant to guard or reduce corrosion in metals. However, over the past several years, a new area of technology has been garnering a great deal of attention and has made use of zinc and its compounds. This is with reference to paper-based microfluidic technology that offers several advantages and that keeps expanding in the amount of applications it covers. In this paper, a review is offered for the applications that have used zinc or zinc compounds in paper-based microfluidic devices.
\end{abstract}

\section{Keywords}

Zinc, Zinc Oxide, Zinc Compounds, Paper-Based Devices, Paper Microfluidics, Low-Cost Platforms, Point-of-Care Diagnostics

\section{Introduction}

Paper has been used for biological and chemical applications for over a century [1] [2]. However, over the past several years, a lot of research and resources have been dedicated to developing new paper-based devices, or improving their performance, for use in a wide range of applications. Paper-based devices have been used for biomedical applications [3], food safety [4], soil analysis [5], water analysis [6] and in many other miscellaneous applications [7]. Muller et al. [8] may have developed the first simple paper-based microfluidic device in 1949 [9] 
[10] [11]; however, it was the Whitesides group in 2007 [12] who gave a renewed view and push for the possible applications of this technology. The main advantages of paper-based microfluidic technology is that these devices are inexpensive, simple, portable and easy to use. However, the most significant advantage is that these devices depend on capillary action to flow the sample across the different sections of the device without a need for a pump, which results in device miniaturization and cost savings. Paper-based microfluidic devices have adapted several of the conventional detection techniques such as colorimetric detection, electrochemical detection, chemiluminescence, fluorescence, mass spectrometry and surface-enhanced Raman spectroscopy [13]. And since zinc and its compounds are utilized in a broad range of applications due to their inherent advantages, it was clear that they were to be used in paper-based microfluidic devices.

Zinc is an essential trace element for the human body since it plays an important role in maintaining cell health and functionality and is crucial for the function of more than 300 enzymes [14] [15]. The human body contains 2 to 3 grams of zinc and requires a daily intake of 10 to $15 \mathrm{mg}$ [16]. Zinc also finds use in many different applications outside of the human body mainly due to its abundance and nontoxicity [17]. Zinc and zinc oxide have been used in the rubber industry, pharmaceutical and cosmetic industries, textile industry, electronics industry, for enhancing filtration, in making batteries, and in many other miscellaneous applications [18] [19] [20] [21]. Zinc oxide is an inorganic material with specific properties that is abundantly produced after silicon dioxide and titanium dioxide [22]. Zinc oxide nanomaterial is stable, safe to human beings and has been categorized by the Food and Drug Administration in the United States as "generally recognized as safe" [23]. It has been vastly used in environmental and medical applications due to its nontoxicity, biosafety, biocompatibility and biodegradability [24]. Textiles have been functionalized by zinc oxide to impart favorable properties such as improved antimicrobial [25], super hydrophobicity [26], self-cleaning [27], thermal insulation [28], UV-absorption [29], and flame retardancy [30]. The cotton fibers in paper-based devices have also been functionalized by zinc oxide to provide or enhance analytical or engineering purposes.

In this review, some of the paper-based microfluidic devices that have used zinc or zinc compounds will be highlighted with the different applications they were used in.

\section{Application of Zinc and Zinc Compounds in Paper-Based Devices}

\subsection{Biomedical}

Zinc and its oxide are biocompatible [31] [32] and have been used in several paper-based devices for different biomedical applications. Li et al. [33] used zinc oxide nanowires for the electrochemical detection of glucose in spiked phosphate-buffered saline and human serum, Figure 1. However, they anticipate that 


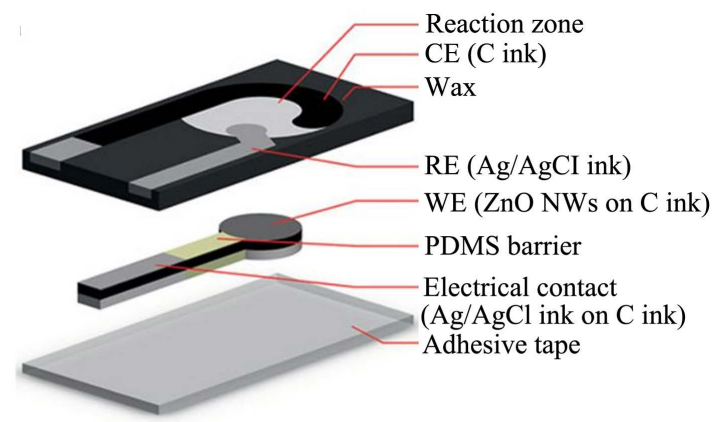

(a)

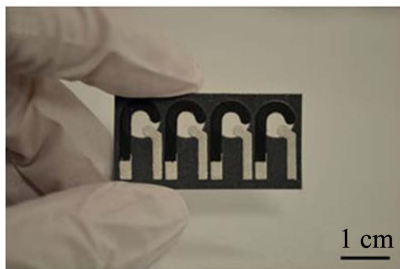

(b)

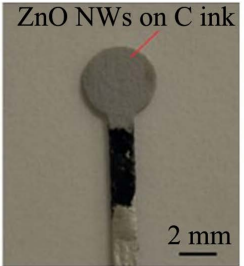

(c)

Figure 1. Paper-based device developed by Li et al. [33] that uses zinc oxide nanowires for the electrochemical detection of glucose.

their device can be used to detect glucose in whole blood if a filtering membrane is added on top of their device's reaction zone to filter out the blood cells. Ferreira et al. [34] used zinc microparticles as the reducing agent in a paper-based device to detect nitrate in human saliva.

Detection of biomarkers using paper-based devices will help in making rapid and informed decisions whether further investigation is needed to determine the health status of an individual. Tiwari et al. [35] functionalized paper with zinc oxide nanorods to preconcentrate myoglobin which is a biomarker for heart disease. Guo et al. [36] developed a paper-based device integrated with zinc oxide nanowires that can detect three cardiac biomarkers which are the human heart-type fatty acid binding protein, cardiac troponin I, and myoglobin, simultaneously. Kong et al. [37] constructed a paper-based device utilizing zinc oxide nanoflowers for the sensitive detection of the biomolecules, L-glutamic acid and L-cysteine. Sun et al. [38] constructed an ultrasensitive electrochemical immunosensor using branched zinc oxide nanorods and porous zinc oxide spheres with gold nanoparticle composite for the detection of $\alpha$-fetoprotein which is a tumor marker used in the diagnosis of hepatocellular carcinoma. Sun et al. [39] used tetra-carboxyl naphthalocyanine zinc sensitized zinc oxide nanorods as photoactive materials in a paper-based photoelectrochemical immunosensor to detect the carcinoembryonic antigen. Liu et al. [40] constructed a paper-based device for the colorimetric determination of the carcinoembryonic antigen based on the intrinsic peroxidase activity of zinc ferrite $\left(\mathrm{ZnFe}_{2} \mathrm{O}_{4}\right)$-multiwalled carbon nanotubes. Wang et al. [41] constructed a hollow-channel paper-based device with CdS quantum dots/reduced graphene oxide/zinc oxide nanorod arrays for the photoelectrochemical detection of multiple microRNAs.

Portable, rapid and sensitive detection of diseases, particularly infectious, 
would play a significant role in decreasing their spread. Li et al. [42] reported on the first microfluidic paper-based origami nanobiosensor that uses zinc oxide nanowires to improve the biosensing performance of detecting the human immunodeficiency virus p24 antigen. Li et al. [43] also used zinc oxide nanowires directly grown on the working electrode of the paper-based device to enhance the detection of the p24 antigen used as a marker for the human immunodeficiency virus and showed that their device was capable of differentiating between concentrations of CR3022, an IgG antibody to the COVID-19 coronavirus. Narang et al. [44] developed a paper-based device incorporating zinc-silver nanoblooms that used cyclic voltammetry for the electrochemical detection of the herpes virus DNA. Kim [45] grew zinc oxide nanorods on cellulose paper and then added gold nanoparticles to these rods so as to enhance the Raman signal in detecting the presence of prenatal diseases and identifying the types of diseases from amniotic fluids.

Finally, it is very useful to have a simple and rapid method for detecting different drugs. Caglayan et al. [46] used a fluorescent coordinatively unsaturated metal complexes based on the zinc II ion to provide an easy-to-detect fluorescence response in the detection of the banned performance-enhancing drug Adrafinil. Narang et al. [47] developed a paper-based device for the detection of methylenedioxymethamphetamine which is an addictive narcotic and a potential neurotoxic agent used as a recreational drug.

\subsection{Environmental Applications}

Paper-based microfluidic devices generally integrate a suitable detection method such as colorimetric, electrochemical or fluorescent techniques for quantitative analysis of nutrients in air, soil, or water [11]. Zinc and its compounds have been used in microfluidic paper-based devices for environmental applications to provide rapid, inexpensive, and quantitative analysis. Nitrate is part of the nitrogen cycle but contributes to water nutrient pollution when present at elevated concentrations [48] [49]. Jayawardane et al. [50] and Charbaji et al. [51] used zinc mircoparticles as a reducing agent in paper-based devices meant for the detection of nitrate in water. The zinc microparticles provided the reduction of nitrate to nitrite needed for its detection by the Griess assay. Figure 2 shows the paper-based device developed by Charbaji et al. for detecting nitrate in water. While Jayawardane et al. created a reduction channel by depositing zinc microparticles on the surface of paper, Charbaji et al. used a newly developed composite material made up of cellulose and zinc microparticles, that they called Zinculose [52], Figure 3, to provide the required reduction step. Zhang et al. [53] used dual-emission manganese-doped $\mathrm{ZnS}$ semiconductor nanocrystals on paper-based test strips for the visual detection of diethylphosphorothioate (organophosphate) residues.

Another environmental application that made use of paper-based microfluidic devices was the detection of pentachlorophenol. Pentachlorophenol is a xenobiotic that enters the environment as a byproduct of various industrial processes 

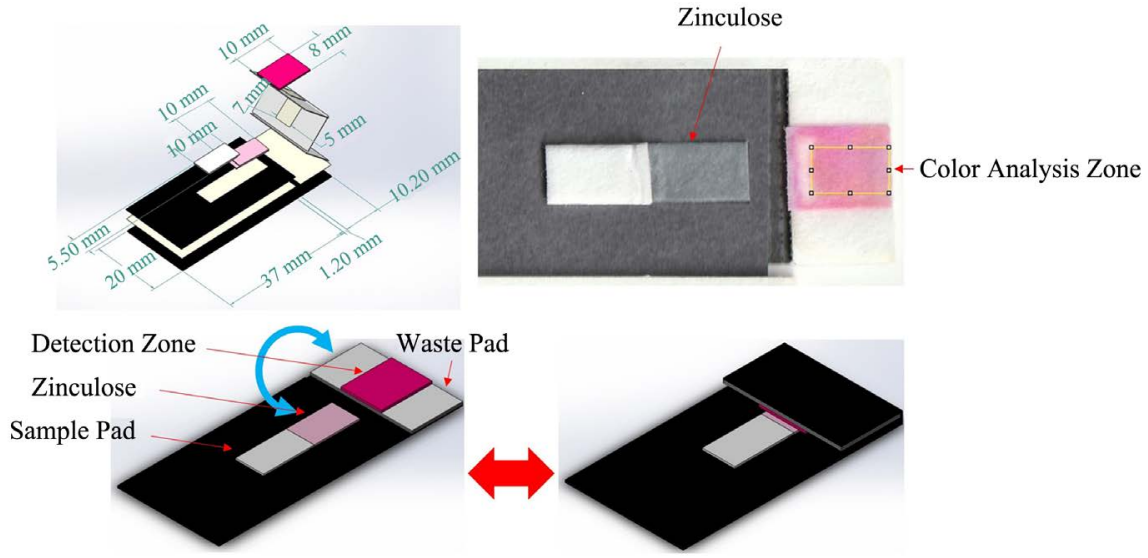

Figure 2. Paper-based device developed by Charbaji et al. [51] for the detection of nitrate in water.

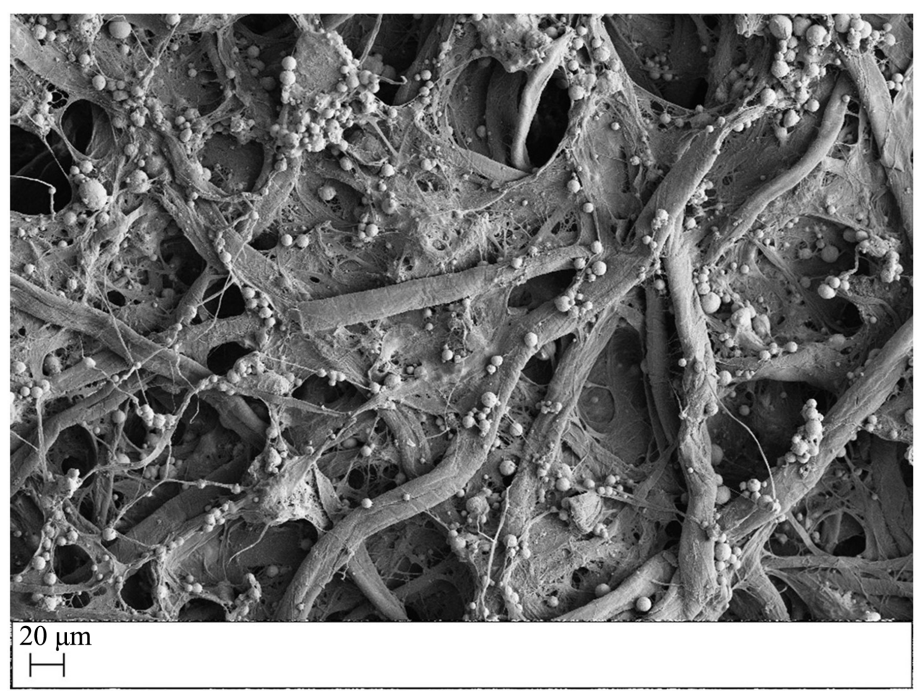

Figure 3. A strip of Zinculose observed using a scanning electron microscope at a magnification of $500 \times[52]$.

and is a cause for great environmental concern. Sun et al. [54] developed a disposable paper-based microfluidic origami device with functionalized zinc oxide nanospheres to detect pentachlorophenol.

Paper-based devices were also used in the detection of gases. Gimenez et al. [55] developed a paper-based oxygen sensor that uses the absorption and desorption of oxygen from the zinc oxide crystals deposited and dried over graphite electrodes drawn on paper for detection. Koga et al. [56] deposited zinc oxide on paper using a two-step paper-making process to create a molecular sensor and they used nitrogen dioxide to test its sensing performance.

\subsection{Food Safety}

Monitoring the quality of agricultural and food products in order to ensure its safety is of utmost importance to safeguard the health and wellbeing of consumers. Developing small-size field deployable sensing systems and combining that 
with state-of-the-art communication techniques is a way to perform qualitative and quantitative multi-component analysis of food products to guarantee its safety [57]. Paper-based devices have many desirable advantages that make them ideal candidates for such an application and they have been demonstrated to be effective in testing the quality of food products [58]. Several paper-based devices utilizing zinc or its compounds have been developed to detect different analytes or toxins in food. Yukird et al. [59] deposited zinc oxide from a solution over 2 detection zones in a paper-based device. The first was a multiwall carbon nanotube electrode and the second was a laser desorption ionization mass spectrometric detection zone. This was meant to improve their sensitivity in detecting "bisphenol A" which is a chemical compound used in the production of food containers.

Chen et al. [60] developed a fluorescence paper-based sensor based on zinc 5, 10, 15, 20-tetra(4-pyridyl)-21H-23H-porphine (nano ZnTPyP) quenching CdTe quantum dots for the detection of three carbamate pesticides which are metolcarb, carbofuran, and carbaryl. Teepo et al. [61] and Ratnarathornet al. [62] used zinc microparticles as the reducing agent in paper-based devices to detect the presence of nitrate in food samples. Figure 4 shows the paper-based device developed by Teepo et al. for detecting nitrate in food samples.

Chen et al. [63] established a high sensitivity paper-based fluorescent sensor to detect L-theanine in tea water which is one of the markers used to evaluate the sweetness and freshness of tea. Their device used CdTe quantum dots/corn carbon dots with nano tetra pyridel-porphine zinc to provide the quenching effect. Another paper-based fluorescent sensor utilizing CdTe quantum dots and spherical nano tetra pyridel-porphine zinc was developed by Chen et al. [64] for the identification and quantitative analysis of caffeine.

\subsection{Miscellaneous Applications}

Although the above sections highlighted microfluidic paper-based devices developed for biomedical, environmental and food safety applications, it's important to mention that there are a lot of other applications that have also used zinc

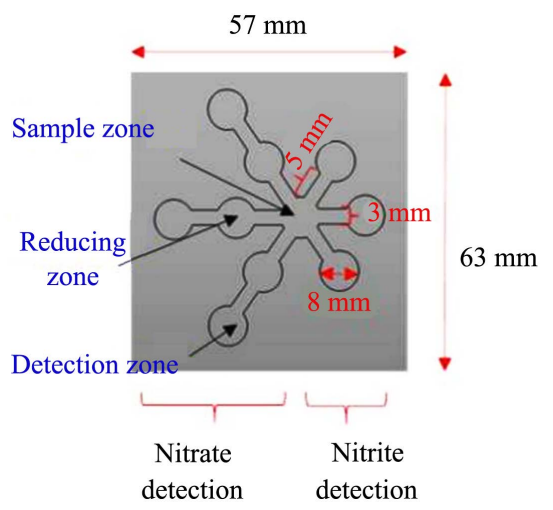

Figure 4. Paper-based device developed by Teepo et al. [61] for the detection of nitrate in food samples. The reducing zone contained zinc dust. 
or its compounds in paper-based devices. This section will refer to some of these paper-based devices used for miscellaneous applications. Song et al. [65] have provided a decent review of paper-based physical sensors utilizing zinc oxide nanostructures up to the year 2017. Therefore, this review looks at papers published from 2017 onwards for paper-based physical sensors utilizing zinc compounds. Wang et al. [66] developed a one-axis piezoelectric accelerometer using zinc oxide nanowires that have been grown on paper, Figure 5. The fabrication of their device is inexpensive and doesn't require sophisticated equipment. Dubourg et al. [67] developed a paper-based ultraviolet sensor utilizing zinc oxide. They used a laser post-processing step to induce significant modifications to the surface morphology and structure of the zinc oxide film. This contributed to the super-hydrophobicity of the printed zinc oxide nanoparticles which reduced humidity interference while enhancing sensitivity for ultraviolet detection.

Some paper-based devices were also developed in order to provide a flexible power-source. Purohit et al. [68] developed microfluidic galvanic and hybrid cells on a single layer of paper. The cell had zinc and copper powder that was painted on the paper to serve as the anode and cathode. Zhang et al. [69] developed a zinc-air battery with zinc foil pasted on titanium foil to act as the anode. Burrola et al. [70] presented an alkaline nickel oxide hydroxide/zinc battery with a zinc anode cut from commercially available sheets.

An interesting paper-based device using zinc oxide was developed by Zhang et al. [71]. They used zinc oxide nanorods to fabricate a flexible light emitting diode on paper and used it as an excitation light source in a multiplexed photoelectrochemical immunosensor.

\section{Challenges and Future Trends}

Zinc, its oxide and compounds have been seeing increased use in paper-based microfluidic devices, and their usage is expected to expand further to encompass more applications. However, some challenges still need to be overcome to ensure repeatability in the preparation of zinc oxide nanoparticles to enable the surface modification of these particles with organic compounds [20]. Also, zinc oxide may need to be combined with other material such as metals, semiconductors,

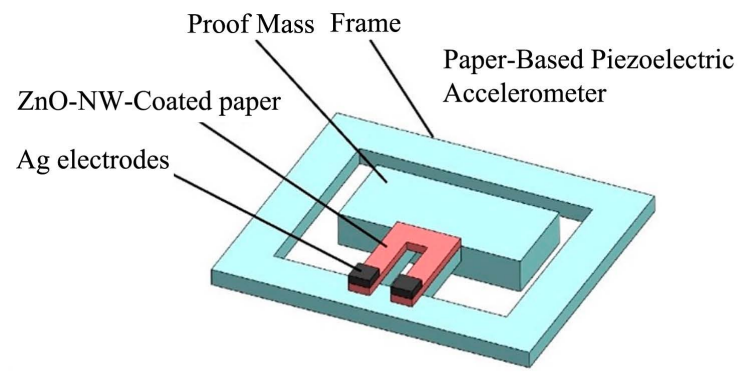

(a)

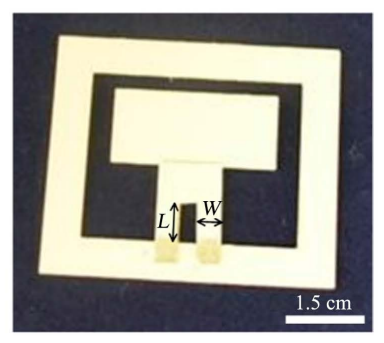

(b)

Figure 5. (a) Schematic of the paper-based one-axis piezoelectric accelerometer developed by Wang et al. [66]; (b) Photograph of the paper-based device. 
and nanocarbons to overcome its limitations such as fast recombination of photogenerated electron-hole pairs, photocorrosion and need for UV light for activation in photocatalytic applications [72].

The development of composite materials using zinc microparticles such as Zinculose [52] will help improve the performance of paper-based devices using zinc microparticles in different applications. As paper-based electrochemical devices start to play a bigger role in the paper-based platform technology, use of zinc oxide is expected to enhance the sensitivity and selectivity of these devices [73]. Zinc oxide has demonstrated effectiveness in biomedical fluorescence assays [74] and will be instrumental when more paper-based devices are developed for fluorescence assays. It is anticipated that the future direction is to develop more paper-based devices for photonic and electronic applications which will use zinc oxide nanoparticles since these particles are ideal for use in the field of photonics, nanoscale electronics and optoelectonics [75] [76].

\section{Conclusion}

Paper-based microfluidic technology is a field that is still developing with frequent new applications and advancements being achieved. Zinc, its oxide and compounds have been utilized in a lot of different applications using paper-based technology. This is the case since zinc, zinc oxide and zinc compounds are inexpensive, biocompatible, non-toxic, environmentally friendly, and have their distinctive physical and chemical properties. They have been widely utilized to improve the properties and performance of cellulose substrates in paper-based analytical devices. In this review, paper-based devices that have used zinc or zinc compounds in different applications were highlighted. These devices were developed for biomedical, environmental, food safety and several other miscellaneous applications. However, it is anticipated that more devices utilizing zinc, its oxide or compounds will be developed in the future. These devices will either improve the performance of the ones that have already been designed before or will be completely novel sensors for new applications.

\section{Acknowledgements}

The authors would like to acknowledge the support from Rhode Island EPSCoR which is funded by the National Science Foundation under Award \#OIA-1655221. The authors would also like to acknowledge the students, research scientists and visiting scholars at the Microfluidics Laboratory at the University of Rhode Island for their help and support.

\section{Conflicts of Interest}

The authors declare no conflict of interest.

\section{References}

[1] Qi, A., Yeo, L., Friend, J. and Ho, J. (2010) The Extraction of Liquid, Protein Mole- 
cules and Yeast Cells from Paper through Surface Acoustic Wave Atomization. Lab on a Chip, 10, 470-476. https://doi.org/10.1039/B915833B

[2] Demirev, P.A. (2013) Dried Blood Spots: Analysis and Applications. Analytical Chemistry, 85, 779-789. https://doi.org/10.1021/ac303205m

[3] Islam, M.N., Ahmed, I., Anik, M.I., et al. (2018) Developing Paper Based Diagnostic Technique to Detect Uric Acid in Urine. Frontiers in Chemistry, 6, 496. https://doi.org/10.3389/fchem.2018.00496

[4] Gao, H., Yan, C., Wu, W. and Li, J. (2020) Application of Microfluidic Chip Technology in Food Safety Sensing. Sensors, 20, 1792.

https://doi.org/10.3390/s20061792

[5] Shriver-Lake, L.C., Zabetakis, D., Dressick, W.J., et al. (2018) Paper-Based Electrochemical Detection of Chlorate. Sensors, 18, 328. https://doi.org/10.3390/s18020328

[6] Heidari-Bafroui, H., Ribeiro, B., Charbaji, A., et al. (2020) Portable Infrared Lightbox for Improving the Detection Limits of Paper-Based Phosphate Devices. Measurement. Journal of the International Measurement Confederation, 173, Article ID: 108607. https://doi.org/10.1016/j.measurement.2020.108607

[7] Singh, A., Lantigua, D., Meka, A., et al. (2018) Paper-Based Sensors: Emerging Themes and Applications. Sensors, 18, 2838. https://doi.org/10.3390/s18092838

[8] Müller, R.H. and Clegg, D.L. (1949) Automatic Paper Chromatography. Analytical Chemistry, 21, 1123-1125. https://doi.org/10.1021/ac60033a032

[9] Soum, V., Park, S., Brilian, A.I., et al. (2019) Programmable Paper-Based Microfluidic Devices for Biomarker Detections. Micromachines, 10, 516.

https://doi.org/10.3390/mi10080516

[10] Li, X., Ballerini, D.R. and Shen, W. (2012) A Perspective on Paper-Based Microfluidics: Current Status and Future Trends. Biomicrofluidics, 6, Article ID: 011301. https://doi.org/10.1063/1.3687398

[11] Manisha, H., Priya Shwetha, P.D. and Prasad, K.S. (2018) Low-Cost Paper Analytical Devices for Environmental and Biomedical Sensing Applications. Springer, Singapore, 315-341. https://doi.org/10.1007/978-981-10-7751-7_14

[12] Martinez, A.W., Phillips, S.T., Butte, M.J. and Whitesides, G.M. (2007) Patterned Paper as a Platform for Inexpensive, Low-Volume, Portable Bioassays. Angewandte Chemie International Edition, 46, 1318-1320. https://doi.org/10.1002/anie.200603817

[13] Liu, S., Su, W. and Ding, X. (2016) A Review on Microfluidic Paper-Based Analytical Devices for Glucose Detection. Sensors (Switzerland), 16, 1-17. https://doi.org/10.3390/s16122086

[14] Stefanidou, M., Maravelias, C., Dona, A. and Spiliopoulou, C. (2006) Zinc: A Multipurpose Trace Element. Archives of Toxicology, 80, 1-9. https://doi.org/10.1007/s00204-005-0009-5

[15] Jansen, J., Karges, W. and Rink, L. (2009) Zinc and Diabetes-Clinical Links and Molecular Mechanisms. Journal of Nutritional Biochemistry, 20, 399-417. https://doi.org/10.1016/j.jnutbio.2009.01.009

[16] Siddiqi, K.S., ur Rahman, A. and Tajuddin, H.A. (2018) Properties of Zinc Oxide Nanoparticles and Their Activity against Microbes. Nanoscale Research Letters, 13, 141. https://doi.org/10.1186/s11671-018-2532-3

[17] López, C.M. and Choi, K.S. (2005) Enhancement of Electrochemical and Photoelectrochemical Properties of Fibrous $\mathrm{Zn}$ and $\mathrm{ZnO}$ Electrodes. Chemical Communica- 
tions, No. 26, 3328-3330. https://doi.org/10.1039/b502238j

[18] Zhang, X.G. (2006) Fibrous Zinc Anodes for High Power Batteries. Journal of Power Sources, 163, 591-597. https://doi.org/10.1016/j.jpowsour.2006.09.034

[19] Caramia, V. and Bozzini, B. (2014) Materials Science Aspects of Zinc-Air Batteries: A Review. Materials for Renewable and Sustainable Energy, 3, Article No. 28. https://doi.org/10.1007/s40243-014-0028-3

[20] Kolodziejczak-Radzimska, A. and Jesionowski, T. (2014) Zinc Oxide-From Synthesis to Application: A Review. Materials (Basel), 7, 2833-2881.

https://doi.org/10.3390/ma7042833

[21] Jakubiak, S., Tomaszewska, J., Jackiewicz, A., et al. (2016) Polypropylene-Zinc Oxide Nanorod Hybrid Material for Applications in Separation Processes. Chemical and Process Engineering-Inzynieria Chemiczna i Procesowa, 37, 393-403. https://doi.org/10.1515/cpe-2016-0032

[22] Mohd Yusof, H., Mohamad, R., Zaidan, U.H. and Abdul Rahman, N.A. (2019) Microbial Synthesis of Zinc Oxide Nanoparticles and Their Potential Application as an Antimicrobial Agent and a Feed Supplement in Animal Industry: A Review. Journal of Animal Science and Biotechnology, 10, 57. https://doi.org/10.1186/s40104-019-0368-Z

[23] Sutradhar, S., Bandyopadhyay, A., Debnath, T., et al. (2019) Effect of Hydrothermal Synthesis on Physical Property Modulation and Biological Activity of $\mathrm{ZnO}$ Nanorods. Materials Research Express, 6, 1250-1257.

https://doi.org/10.1088/2053-1591/ab4dd0

[24] Zhang, Y., Ram, M.K., Stefanakos, E.K. and Goswami, D.Y. (2012) Synthesis, Characterization, and Applications of $\mathrm{ZnO}$ Nanowires. Journal of Nanomaterials, 2012, Article ID: 624520. https://doi.org/10.1155/2012/624520

[25] Verbič, A., Gorjanc, M. and Simončič, B. (2019) Zinc Oxide for Functional Textile Coatings: Recent Advances. Coatings, 9, 550.

https://doi.org/10.3390/coatings9090550

[26] Ghasemi, N., Seyfi, J. and Asadollahzadeh, M.J. (2018) Imparting Superhydrophobic and Antibacterial Properties onto the Cotton Fabrics: Synergistic Effect of Zinc Oxide Nanoparticles and Octadecanethiol. Cellulose, 25, 4211-4222. https://doi.org/10.1007/s10570-018-1837-9

[27] Zhu, C., Shi, J., Xu, S., et al. (2017) Design and Characterization of Self-Cleaning Cotton Fabrics Exploiting Zinc Oxide Nanoparticle-Triggered Photocatalytic Degradation. Cellulose, 24, 2657-2667. https://doi.org/10.1007/s10570-017-1289-7

[28] Wang, W., Liang, Y., Yang, Z., et al. (2019) Construction of Ultraviolet Protection, Thermal Insulation, Superhydrophobic and Aromatic Textile with Al-Doped ZnOEmbedded Lemon Microcapsule Coatings. Textile Research Journal, 89, 3860-3870. https://doi.org/10.1177/0040517518824842

[29] Yadav, A., Prasad, V., Kathe, A.A., et al. (2006) Functional Finishing in Cotton Fabrics Using Zinc Oxide Nanoparticles. Bulletin of Materials Science, 29, 641-645. https://doi.org/10.1007/s12034-006-0017-y

[30] El-Hady, M.M.A., Farouk, A. and Sharaf, S. (2013) Flame Retardancy and UV Protection of Cotton Based Fabrics Using Nano $\mathrm{ZnO}$ and Polycarboxylic Acids. Carbohydrate Polymers, 92, 400-406. https://doi.org/10.1016/j.carbpol.2012.08.085

[31] Chen, Y., Huang, P., Chen, H., et al. (2018) Assessment of the Biocompatibility and Biological Effects of Biodegradable Pure Zinc Material in the Colorectum. ACS Biomaterials Science \& Engineering, 4, 4095-4103. https://doi.org/10.1021/acsbiomaterials.8b00957 
[32] Jiang, J., Pi, J. and Cai, J. (2018) The Advancing of Zinc Oxide Nanoparticles for Biomedical Applications. Bioinorganic Chemistry and Applications, 2018, Article ID: 1062562. https://doi.org/10.1155/2018/1062562

[33] Li, X., Zhao, C. and Liu, X. (2015) A Paper-Based Microfluidic Biosensor Integrating Zinc Oxide Nanowires for Electrochemical Glucose Detection. Microsystems \& Nanoengineering, 1, 1. https://doi.org/10.1038/micronano.2015.14

[34] Ferreira, F.T.S.M., Mesquita, R.B.R. and Rangel, A.O.S.S. (2020) Novel Microfluidic Paper-Based Analytical Devices ( $\mu$ PADs) for the Determination of Nitrate and Nitrite in Human Saliva. Talanta, 219, Article ID: 121183. https://doi.org/10.1016/j.talanta.2020.121183

[35] Tiwari, S., Vinchurkar, M., Rao, V.R. and Garnier, G. (2017) Zinc Oxide Nanorods Functionalized Paper for Protein Preconcentration in Biodiagnostics. Scientific Reports, 7, Article No. 43905. https://doi.org/10.1038/srep43905

[36] Guo, X., Zong, L., Jiao, Y., et al. (2019) Signal-Enhanced Detection of Multiplexed Cardiac Biomarkers by a Paper-Based Fluorogenic Immunodevice Integrated with Zinc Oxide Nanowires. Analytical Chemistry, 91, 9300-9307.

https://doi.org/10.1021/acs.analchem.9b02557

[37] Kong, Q., Wang, Y., Zhang, L., et al. (2018) Highly Sensitive Microfluidic Paper-Based Photoelectrochemical Sensing Platform Based on Reversible Photo-Oxidation Products and Morphology-Preferable Multi-Plate ZnO Nanoflowers. Biosensors and Bioelectronics, 110, 58-64. https://doi.org/10.1016/j.bios.2018.03.050

[38] Sun, G., Yang, H., Zhang, Y., et al. (2015) Branched Zinc Oxide Nanorods Arrays Modified Paper Electrode for Electrochemical Immunosensing by Combining Biocatalytic Precipitation Reaction and Competitive Immunoassay Mode. Biosensors and Bioelectronics, 74, 823-829. https://doi.org/10.1016/j.bios.2015.07.057

[39] Sun, G., Wang, P., Zhu, P., et al. (2014) A Near-Infrared Light Photoelectrochemical Immunosensor Based on a Au-Paper Electrode and Naphthalocyanine Sensitized ZnO Nanorods. Journal of Materials Chemistry B, 2, 4811-4817. https://doi.org/10.1039/C4TB00623B

[40] Liu, W., Yang, H., Ding, Y., et al. (2014) Paper-Based Colorimetric Immunosensor for Visual Detection of Carcinoembryonic Antigen Based on the High Peroxidase-Like Catalytic Performance of $\mathrm{ZnFe}_{2} \mathrm{O}_{4}$-Multiwalled Carbon Nanotubes. Analyst, 139, 251-258. https://doi.org/10.1039/C3AN01569F

[41] Wang, Y., Zhang, L., Kong, Q., et al. (2018) Time-Resolution Addressable Photoelectrochemical Strategy Based on Hollow-Channel Paper Analytical Devices. Biosensors and Bioelectronics, 120, 64-70. https://doi.org/10.1016/j.bios.2018.08.028

[42] Li, X. and Liu, X. (2016) A Microfluidic Paper-Based Origami Nanobiosensor for Label-Free, Ultrasensitive Immunoassays. Advanced Healthcare Materials, 5, 13261335. https://doi.org/10.1002/adhm.201501038

[43] Li, X., Qin, Z., Fu, H., et al. (2021) Enhancing the Performance of Paper-Based Electrochemical Impedance Spectroscopy Nanobiosensors: An Experimental Approach. Biosensors and Bioelectronics, 177, Article ID: 112672. https://doi.org/10.1016/j.bios.2020.112672

[44] Narang, J., Singhal, C., Mathur, A., et al. (2018) Portable Bioactive Paper Based Genosensor Incorporated with $\mathrm{Zn}-\mathrm{Ag}$ Nanoblooms for Herpes Detection at the Point-of-Care. International Journal of Biological Macromolecules, 107, 2559-2565. https://doi.org/10.1016/j.ijbiomac.2017.10.146

[45] Kim, W., Lee, S.H., Kim, J.H., et al. (2018) Paper-Based Surface-Enhanced Raman 
Spectroscopy for Diagnosing Prenatal Diseases in Women. ACS Nano, 12, 71007108. https://doi.org/10.1021/acsnano.8b02917

[46] Caglayan, M.G., Sheykhi, S., Mosca, L. and Anzenbacher, P. (2016) Fluorescent Zinc and Copper Complexes for Detection of Adrafinil in Paper-Based Microfluidic Devices. Chemical Communications, 52, 8279-8282. https://doi.org/10.1039/C6CC03640F

[47] Narang, J., Singhal, C., Khanuja, M., et al. (2017) Hydrothermally Synthesized Zinc Oxide Nanorods Incorporated on Lab-on-Paper Device for Electrochemical Detection of Recreational Drug. Artificial Cells Nanomedicine and Biotechnology, 46, 1-8. https://doi.org/10.1080/21691401.2017.1381614

[48] World Health Organization (2017) Guidelines for Drinking-Water Quality. 4th Edition, Incorporating the 1st Addendum.

https://www.who.int/publications/i/item/9789241549950

[49] U.S. Geological Survey Nitrogen and Water. https://www.usgs.gov/special-topic/water-science-school/science/nitrogen-and-wate r?qt-science_center_objects=0\#qt-science_center_objects

[50] Jayawardane, B.M., Wei, S., McKelvie, I.D. and Kolev, S.D. (2014) Microfluidic Paper-Based Analytical Device for the Determination of Nitrite and Nitrate. Analytical Chemistry, 86, 7274-7279. https://doi.org/10.1021/ac5013249

[51] Charbaji, A., Heidari-Bafroui, H., Anagnostopoulos, C. and Faghri, M. (2020) A New Paper-Based Microfluidic Device for Improved Detection of Nitrate in Water. Sensors, 21, 102. https://doi.org/10.3390/s21010102

[52] Charbaji, A., Smith, W., Anagnostopoulos, C. and Faghri, M. (2020) Zinculose: A New Fibrous Material with Embedded Zinc Particles. Engineering Science and Technology, an International Journal, 24, 571-578. https://doi.org/10.1016/j.jestch.2020.09.005

[53] Zhang, K., Yu, T., Liu, F., et al. (2014) Selective Fluorescence Turn-On and Ratiometric Detection of Organophosphate Using Dual-Emitting Mn-Doped ZnS Nanocrystal Probe. Analytical Chemistry, 86, 11727-11733. https://doi.org/10.1021/ac503134r

[54] Sun, G., Wang, P., Ge, S., et al. (2014) Photoelectrochemical Sensor for Pentachlorophenol on Microfluidic Paper-Based Analytical Device Based on the Molecular Imprinting Technique. Biosensors and Bioelectronics, 56, 97-103. https://doi.org/10.1016/j.bios.2014.01.001

[55] Gimenez, A.J., Yáñez-Limón, J.M. and Seminario, J.M. (2013) ZnO-Cellulose Composite for UV Sensing. IEEE Sensors Journal, 13, 1301-1306. https://doi.org/10.1109/JSEN.2012.2231067

[56] Koga, H., Nagashima, K., Huang, Y., et al. (2019) Paper-Based Disposable Molecular Sensor Constructed from Oxide Nanowires, Cellulose Nanofibers, and Pencil-Drawn Electrodes. ACS Applied Materials \& Interfaces, 11, 15044-15050. https://doi.org/10.1021/acsami.9b01287

[57] Galstyan, V., Bhandari, M., Sberveglieri, V., et al. (2018) Metal Oxide Nanostructures in Food Applications: Quality Control and Packaging. Chemosensors, 6, 16. https://doi.org/10.3390/chemosensors6020016

[58] Li, Z., Zhu, Y., Zhang, W., et al. (2017) A Low-Cost and High Sensitive Paper-Based Microfluidic Device for Rapid Detection of Glucose in Fruit. Food Analytical Methods, 10, 666-674. https://doi.org/10.1007/s12161-016-0626-Z

[59] Yukird, J., Soum, V., Kwon, O.S., et al. (2020) 3D Paper-Based Microfluidic Device: A Novel Dual-Detection Platform of Bisphenol A. Analyst, 145, 1491-1498. 
https://doi.org/10.1039/C9AN01738K

[60] Chen, H., Hu, O., Fan, Y., et al. (2020) Fluorescence Paper-Based Sensor for Visual Detection of Carbamate Pesticides in Food Based on CdTe Quantum Dot and Nano Zntpyp. Food Chemistry, 327, Article ID: 127075. https://doi.org/10.1016/j.foodchem.2020.127075

[61] Teepoo, S., Arsawiset, S. and Chanayota, P. (2019) One-Step Polylactic Acid Screen-Printing Microfluidic Paper-Based Analytical Device: Application for Simultaneous Detection of Nitrite and Nitrate in Food Samples. Chemosensors, 7, 44. https://doi.org/10.3390/chemosensors7030044

[62] Ratnarathorn, N. and Dungchai, W. (2020) Paper-Based Analytical Device (PAD) for the Determination of Borax, Salicylic Acid, Nitrite, and Nitrate by Colorimetric Methods. Journal of Analytical Chemistry, 75, 487-494.

https://doi.org/10.1134/S1061934820040127

[63] Chen, H., Wei, L., Guo, X., et al. (2020) Determination of L-Theanine in Tea Water Using Fluorescence-Visualized Paper-Based Sensors Based on CdTe Quantum Dots/Corn Carbon Dots and Nano-Porphyrin with Chemometrics. Journal of the Science of Food and Agriculture, 101, 2552-2560. https://doi.org/10.1002/jsfa.10882

[64] Chen, H., Liu, R., Guo, X., et al. (2021) Visual Paper-Based Sensor for the Highly Sensitive Detection of Caffeine in Food and Biological Matrix Based on CdTe-Nano ZnTPyP Combined with Chemometrics. Microchimica Acta, 188, 1-11. https://doi.org/10.1007/s00604-020-04663-3

[65] Song, P., Wang, Y.-H. and Liu, X. (2017) Flexible Physical Sensors Made from Paper Substrates Integrated with Zinc Oxide Nanostructures. Flexible and Printed Electronics, 2, Article ID: 034001. https://doi.org/10.1088/2058-8585/aa765d

[66] Wang, Y.-H., Song, P., Li, X., et al. (2018) A Paper-Based Piezoelectric Accelerometer. Micromachines, 9, 19. https://doi.org/10.3390/mi9010019

[67] Dubourg, G., Radović, M. and Vasić, B. (2021) Laser-Tunable Printed ZnO Nanoparticles for Paper-Based UV Sensors with Reduced Humidity Interference. Nanomaterials, 11, 80. https://doi.org/10.3390/nano11010080

[68] Purohit, K.H., Emrani, S., Rodriguez, S., et al. (2016) A Microfluidic Galvanic Cell on a Single Layer of Paper. Journal of Power Sources, 318, 163-169. https://doi.org/10.1016/j.jpowsour.2016.03.109

[69] Zhang, H., Zhang, B., Yang, Y., et al. (2021) A High Power Density Paper-Based Zinc-Air Battery with a Hollow Channel Structure. Chemical Communications, 57, 1258-1261. https://doi.org/10.1039/D0CC07687B

[70] Burrola, S., Horii, M., Gonzalez-Guerrero, M.J., et al. (2020) Production of a $\mathrm{NiO} / \mathrm{Al}$ Primary Battery Employing Powder-Based Electrodes. Electrophoresis, 41, 131-136. https://doi.org/10.1002/elps.201900255

[71] Zhang, Y., Ge, L., Li, M., et al. (2014) Flexible Paper-Based ZnO Nanorod LightEmitting Diodes Induced Multiplexed Photoelectrochemical Immunoassay. Chemical Communications, 50, 1417-1419. https://doi.org/10.1039/C3CC48421A

[72] Albiter, E., Barrera-Andrade, J.M., Rojas-García, E. and Valenzuela, M.A. (2018) Recent Advances of Nanocarbon-Inorganic Hybrids in Photocatalysis. In: Nanocarbon and Its Composites. Preparation, Properties and Applications, Elsevier, Amsterdam, 521-588. https://doi.org/10.1016/B978-0-08-102509-3.00017-1

[73] Puiu, M., Mirceski, V. and Bala, C. (2021) Paper Based Diagnostic Platforms and Devices. Current Opinion in Electrochemistry, 27, Article ID: 100726.

https://doi.org/10.1016/j.coelec.2021.100726

[74] Hahm, J.I. (2014) Zinc Oxide Nanomaterials for Biomedical Fluorescence Detec- 
tion. Journal of Nanoscience and Nanotechnology, 14, 475-486.

https://doi.org/10.1166/jnn.2014.9099

[75] Yi, G.C., Wang, C. and Park, W.I. (2005) ZnO Nanorods: Synthesis, Characterization and Applications. Semiconductor Science and Technology, 20, 22-34. https://doi.org/10.1088/0268-1242/20/4/003

[76] Anbuselvan, D., Nilavazhagan, S., Santhanam, A., et al. (2021) Room Temperature Ferromagnetic Behavior of Nickel-Doped Zinc Oxide Dilute Magnetic Semiconductor for Spintronics Applications. Physica E: Low-Dimensional Systems and Nanostructures, 129, Article ID: 114665. https://doi.org/10.1016/j.physe.2021.114665 